\section{Antibiotics v2.0: computational and synthetic biology approaches to combat antibiotic resistance}
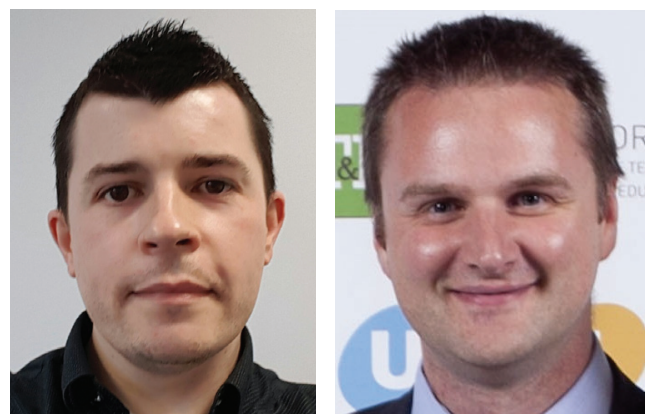

“...only two new classes of antibiotics have reached the market since the 1960's."

Eamonn P Culligan ${ }^{*, 1} \&$ Roy D Sleator ${ }^{* *, 1}$

First draft submitted: 17 January 2017; Accepted for publication: 17 January 2017; Published online: 3 March 2017

Antibiotic-resistant pathogens cause significant morbidities and mortalities each year, killing approximately 700,000 people globally; a figure that is predicted to increase to 10 million by 2050 [1]. Treatment of bacterial infections is increasingly hampered by rapidly growing rates of antibiotic resistance, which is exacerbated by the combined overuse and misuse of antibiotics in medicine and agriculture. A recent declaration following a meeting of the UN General Assembly stated, "antibiotic resistance is the greatest and most urgent global risk, requiring increased attention and coherence at the international, national and regional levels" [2]. There, thus, exists an urgent need for new antimicrobials; an imperative reinforced by the fact that only two new classes of antibiotics have reached the market since the 1960's [3]. Recent reports of bacteria isolated from animals and humans which are resistant to the so-called last-line antibiotic, colistin, is a worrying development, especially given that the resistance determinant, $m c r-1$, is plasmid-encoded and thus mobile [4,5]. In the last year alone, numerous reports have emerged confirming the spread of $m c r-1$ globally [6], and in some cases, in combination with extended-spectrum $\beta$-lactamase production [7]. Colistin resistance is not a new phenomenon, but the fact that the $m c r-1$ gene is horizontally transmissible, highlights a continuously evolving landscape in antimicrobial resistance. This is in addition to the identification of novel antimicrobial resistance genes and atypical mechanisms of resistance, for example, a novel tetracycline efflux system from the oral metagenome [8] and intracellular antibiotic inactivation [9]. Even more worryingly, a carbapenem-resistant Enterobacteriaceae Klebsiella pneumoniae isolate resistant to all available antibiotics in the USA was linked to a fatal infection in a patient who had recently returned from India [10]. The strain displayed panresistance to 26 antibiotics, including all aminoglycosides and polymyxins, yet was mor-1-negative. Taken together, these reports highlight the hastening slide toward the emergence of pan-resistant microbial infections.

\section{KEYWORDS}

- antibiotic resistance $\bullet$ antimicrobial

- bacteriocin • bioinformatics

- biosynthetic gene cluster $\bullet$ colistin

- mcr-1 • multi-drug resistance

- synthetic biology • synthetic DNA 
“The potential to identify novel biosynthetic gene clusters and products thereof is enormous..."
Proposals on coping with the crisis, such as the comprehensive 'O'Neill Report' on antimicrobial resistance [1], details specific intervention strategies and states that, "many known avenues of research for AMR have failed; we need to go back to the drawing board and look for new ones." In line with this call to arms, one promising approach is the use of bioinformatics in combination with synthetic biology and DNA and peptide synthesis to identify and produce novel antimicrobials, by mining genome and metagenome sequence data for biosynthetic gene clusters (BGCs).

BGCs encode antimicrobial compounds, such as polyketide antibiotics, nonribosomal peptides, alkaloids, saccharides, terpenoids and bacteriocins (ribosomally synthesized and post-translationally modified peptides) [11] which have the potential to control pathogenic microorganisms [12]. Approximately $70 \%$ of antimicrobials currently used in medicine are products or derivatives of bacterial and fungal secondary metabolism. The vast amounts of genome and metagenome sequence data generated provide an almost limitless supply of genes to be 'mined' for novel antimicrobials. A large proportion of such microorganisms are yet to be cultured in the laboratory, but crucially, advances in DNA sequencing and synthesis have enabled researchers to access the enormous diversity of antimicrobials produced, providing a means to study these organisms without the need for laboratory culture.

For example, a recent study identified more than 14,000 BGCs in the human microbiome, of which a subset of approximately 3000 were examined in more detail [13], while dozens of bacteriocin gene clusters were identified by analyzing the Human Microbiome Project gut metagenome dataset [14]. Numerous bioinformatic databases and algorithms exist to identify such BGCs (detailed in Medema and Fischbach [15]). Encouragingly, a number of studies have begun to take this approach a step further than highthroughput bioinformatic screening and actually synthesize antimicrobials (named synthetic-bioinformatic natural products) based on primary sequence data alone. Focusing on nonribosomal peptide synthetases and taking advantage of advanced algorithms for predicting the chemical structure from sequence data, allowed Chu et al. to synthesize novel antimicrobials (humimycins) which were active against methicillin-resistant Staphylococcus aureus clinical isolates and which enhanced the effect of certain $\beta$-lactam antibiotics and increased murine survival following infection [16]. This is a powerful approach, which holds significant future promise as it overcomes: the need to culture the producing organism, the fact that many BGCs remain silent under laboratory conditions and gene expression issues if using a heterologous host. The authors state that there are $>1500$ similar gene clusters suitable for this approach, a number which is certain to increase exponentially as more microbial genomes are sequenced (it is conservatively estimated that $>1$ million bacterial and fungal genomes will have been sequenced over the next 10 years) [15], particularly from novel or underrepresented phyla. Using a similar approach, two antimicrobials were identified and synthesized; one with antibacterial and one with antifungal activity following a screen against ESKAPE (Enterococcus faecium, S. aureus, K. pneumoniae, Acinetobacter baumannii, Pseudomonas aeruginosa and Enterobacter species) pathogens and Candida albicans [17]

The potential to identify novel BGCs and products thereof is enormous, especially when one considers that an estimated $99 \%$ of microbes are as yet uncultured [18]. Even among the best-studied natural product producers, the Streptomycetes, it is estimated that only $5 \%$ of their predicted 150,000 natural products have been characterized to date $[19,20]$. Improved bioinformatic algorithms, advances in long-read sequencing (e.g., PacBio and Oxford Nanopore) and singlecelled genomics, coupled with decreases in the cost of DNA sequencing and synthesis will provide a means to access the enormous diversity of BGCs and develop novel antimicrobials. This will also bypass the need to culture the producing organism or heterologously express BGCs by synthesizing the compound directly. In addition to this, synthetic biology is likely to play a key role in the design, modification and production of natural products from BCGs [19]. Modifying or creating new genetic circuits to improve gene expression and to introduce strong promoters and regulators may be helpful to produce larger, more intricate compounds, which currently are too complex for direct synthesis.

Against this backdrop, a number of collaborative projects, as part of the National Microbiome Initiative, are investing heavily to identify novel antimicrobials as part of natural product discovery pipelines [21]. Additionally, the Bill and Melinda Gates Foundation has provided \$17 million USD start-up funding 
for Lodo Therapeutics in a similar vein [22]. Such continued investment is vital to drive this area of research forward, to identify, characterize and produce novel antimicrobials and ultimately to translate such discoveries to viable clinical therapeutics effective against emerging multidrug-resistant pathogens.

In the words of Winston Churchill, in the context of a different global conflict, "This is not the end. It is not even the beginning of the end. But it is, perhaps, the end of the beginning."

\section{Financial \& competing interests disclosure}

EP Culligan is funded by an Irish Research Council Government of Ireland Postdoctoral Fellowship (GOIPD/2015/53). RD Sleator is coordinator of the EUFP7 project ClouDx-i. The authors have no other relevant affiliations or financial involvement with any organization or entity with a financial interest in or financial conflict with the subject matter or materials discussed in the manuscript apart from those disclosed.

No writing assistance was utilized in the production of this manuscript.

\section{References}

1 O'Neill J. Tackling drug-resistant infections globally: final report and recommendations. Rev. Antimicrob. Res. 82 (2016).

2 United Nations. Draft political declaration of the high-level meeting of the General Assembly on antimicrobial resistance (2016). https://www.un.org/pga/71/wp

3 Coates AR, Halls G, Hu Y. Novel classes of antibiotics or more of the same? Br. J. Pharmacol. 163 (1), 184-194 (2011).

4 Huang L, Wang X, Feng Y, Xie Y, Xie L, Zong Z. First identification of an IMI-1 carbapenemase-producing colistin-resistant Enterobacter cloacae in China. Ann. Clin. Microbiol. Antimicrob. 14, 51 (2015).

5 Liu YY, Wang Y, Walsh TR et al. Emergence of plasmid-mediated colistin resistance mechanism MCR-1 in animals and human beings in China: a microbiological and molecular biological study. Lancet Infect. Dis. 16(2), 161-168 (2016).

6 Schwarz S, Johnson AP. Transferable resistance to colistin: a new but old threat. J. Antimicrob. Chemother. 71(8), 2066-2070 (2016).

7 Robin F, Beyrouthy R, Colot J et al. MCR-1 in ESBL-producing Escherichia coli responsible for human infections in New Caledonia. J. Antimicrob. Chemother. doi:10.1093/jac/dkw508 (2016) (Epub ahead of print).
8 Reynolds LJ, Roberts AP, Anjum MF. Efflux in the oral metagenome: the discovery of a novel tetracycline and tigecycline abc transporter. Front. Microbiol. 7, 1923 (2016).

9 Sorg RA, Lin L, Van Doorn GS et al. Collective resistance in microbial communities by intracellular antibiotic deactivation. PLoS Biol. 14(12), e2000631 (2016).

10 Chen L, Todd R, Kiehlbauch J, Walters M, Kallen A. Notes from the field: pan-resistant New Delhi metallo-beta-lactamase-producing Klebsiella pneumoniae - Washoe County, Nevada, 2016. MMWR Morb. Mortal. Wkly Rep. 66(1), 33 (2017).

11 Cotter PD, Ross RP, Hill C. Bacteriocins a viable alternative to antibiotics? Nat. Rev. Microbiol. 11(2), 95-105 (2013).

12 Medema MH, Kottmann R, Yilmaz P et al. Minimum information about a biosynthetic gene cluster. Nat. Chem. Biol. 11(9), 625-631 (2015).

13 Donia MS, Cimermancic P, Schulze CJ et al. A systematic analysis of biosynthetic gene clusters in the human microbiome reveals a common family of antibiotics. Cell 158(6), 1402-1414 (2014).

14 Walsh CJ, Guinane CM, Hill C, Ross RP, O'Toole PW, Cotter PD. In silico identification of bacteriocin gene clusters in the gastrointestinal tract, based on the Human Microbiome Project's reference genome database. BMC Microbiol. 15, 183 (2015).
15 Medema MH, Fischbach MA. Computational approaches to natural product discovery. Nat. Chem. Biol. 11(9), 639-648 (2015).

16 Chu J, Vila-Farres X, Inoyama D et al. Discovery of MRSA active antibiotics using primary sequence from the human microbiome. Nat. Chem. Biol. 12(12), 1004-1006 (2016).

17 Vila-Farres $\mathrm{X}$, Chu J, Inoyama D et al. Antimicrobials inspired by nonribosomal peptide synthetase gene clusters. J. Am. Chem. Soc. doi:10.1021/jacs.6b11861 (2017) (Epub ahead of print).

18 Streit WR, Schmitz RA. Metagenomics--the key to the uncultured microbes. Curr. Opin. Microbiol. 7(5), 492-498 (2004).

19 Smanski MJ, Zhou H, Claesen J, Shen B, Fischbach MA, Voigt CA. Synthetic biology to access and expand nature's chemical diversity. Nat. Rev. Microbiol. 14(3), 135-149 (2016).

20 Watve MG, Tickoo R, Jog MM, Bhole BD. How many antibiotics are produced by the genus Streptomyces? Arch. Microbiol. 176(5), 386-390 (2001).

21 The White House Office of Science and Technology Policy. Announcing the National Microbiome Initiative (2016). https://obamawhitehouse.archives.gov/

22 Lodo Therapeutics - Mining bacterial genomes for novel natural products. http://cen.acs.org/articles/94/i43 